\title{
Comparação do efeito do ácido etilenodiaminotetracético (EDTA) e da heparina sobre os eritrócitos de avestruzes (Struthio camelus L.)
}

\author{
Antonio José Sabino ${ }^{1}$, Silvia Cellone Trevelin², Paulo César Ciarlini ${ }^{3}$
}

\begin{abstract}
RESUMO
Anticoagulantes com diferentes formas de ação têm sido utilizados na hematologia aviária. Entretanto, poucos estudos foram publicados sobre seu efeito e sobre os parâmetros hematológicos dos avestruzes. Visando a preencher essa lacuna, realizou-se um estudo em que foi comparado o efeito de dois anticoagulantes de rotina (EDTA - $2 \mathrm{mg} / \mathrm{mL}$ sangue e da Heparina -10U/mL sangue) sobre os eritrócitos de avestruzes. Para tanto, foram avaliados a integridade, o volume globular e a morfometria dos eritrócitos de amostras sanguíneas de 20 aves, tratadas com ambos os anticoagulantes. A integridade eritrocitária foi estimada pelo grau de hemoglobina livre no plasma, sendo o volume globular obtido pelo método de micro-hematócrito e os dados morfométricos (área, diâmetro maior e menor) calculados automaticamente em sistema computadorizado de imagem digital de alta resolução. A hemólise estimada pela dosagem de hemoglobina plasmática, o volume globular, a área e o diâmetro máximo dos eritrócitos foram significativamente maiores nas amostras tratadas com EDTA. Para melhor preservar a integridade e a morfometria dos eritrócitos, concluiuse que a heparina é um anticoagulante mais adequado que o EDTA para avaliar as discrasias eritrocitárias dos avestruzes.
\end{abstract}

Palavras- chave: Hemácias, interferência de anticoagulante, morfometria eritrocitária, técnica de colheita, hematologia aviária.

\section{ABSTRACT}

\section{Effect of ethylenediaminetetraacetic acid (EDTA) and heparin on erythrocytes of ostriches (Struthio camelus L.)}

Anticoagulants with different modes of action have been used in avian hematology, however, few studies on their effects on the hematological parameters of ostriches were published. Seeking to fill this gap, the effect of two routine anticoagulants (EDTA - $2 \mathrm{mg} / \mathrm{mL}$ blood and Heparin - $10 \mathrm{U} / \mathrm{mL}$ blood) on erythrocytes of ostriches was compared. Blood samples of 20 ostriches, treated with both anticoagulants were evaluated for integrity, globular volume and morphometry of the erythrocytes. The erythrocyte integrity was estimated by the concentration of free hemoglobin in plasma. The globular volume was obtained by the method of microhematocrit and morphometric data (area, bigger and smaller diameters) were automatically calculated by a computerized system for high resolution digital image. Hemolysis estimated by plasma hemoglobin, packed cell volume, area

Recebido para publicação em agosto de 2008 e aprovado em março de 2010

${ }^{1}$ Médico Veterinário. Laborvet Laboratório Veterinário de Análises e Tecnologia Ltda,

Rua Rubião Junior, 2401, Boa Vista, 15025-080, São Jose do Rio Preto, São Paulo, Brasil.

${ }^{2}$ Médica veterinária. Universidade de São Paulo, Faculdade de Medicina de Ribeirão Preto, Departamento de Farmacologia, Avenida Bandeirantes, 3900, Monte Alegre, 14049900, Ribeirão Preto, São Paulo, Brasil.

${ }^{3}$ Médico Veterinário, Doutor. Universidade Estadual Paulista “Julio de Mesquita Filho”, Unesp, Campus de Araçatuba, Departamento de Clínica, Cirurgia e Reprodução Animal, Rua Clóvis Pestana, 793, 16050-680, Araçatuba, São Paulo, Brasil. ciarlini@fmva.unesp.br * Autor para correspondência. 
and maximum diameter of erythrocytes were significantly higher in the samples treated with EDTA. To better preserve the integrity and morphometry of erythrocytes, it was concluded that the heparin rather than EDTA is a more appropriated anticoagulant to evaluate erythrocyte dyscrasia in ostriches.

Key words: Anticoagulant interference, avian hematology, blood collection techniques, erythrocyte morphometry, red blood cells (erythrocytes).

\section{INTRODUÇÃO}

A demanda mundial por produtos de alto valor agregado proporcionou a popularização da criação de avestruzes em diversos países, cujo rebanho mundial, explorado zootecnicamente, chega a aproximadamente quatro milhões de aves. O Brasil apresenta um plantel em formação na primeira fase de produção, com cerca de dez anos e aproximadamente 200 mil aves, distribuídas em várias regiões do país (ACAB, 2005). Estima-se que a industrialização se inicie nos próximos três anos. $\mathrm{O}$ interesse na patologia clínica desses animais determinou a necessidade de estabelecer perfis hematológicos para auxiliar no diagnóstico das doenças.

A Heparina possui ação antitrombínica, impedindo a coagulação, enquanto o ácido etilenodiaminotetracético (EDTA) é um composto orgânico que age formando complexos muito estáveis com diversos íons metálicos. Em razão dessa propriedade, o sal EDTA é usado como anticoagulante do sangue, pois quela os íons de cálcio, que promovem a coagulação sanguínea (Jain, 1993).

Pennock \& Jones (1966) compararam o efeito do EDTA e da Heparina sobre os índices hematimétricos do sangue humano e demonstraram que esse sal produz valores de volume corpuscular médio (VCM) e de concentração da hemoglobina corpuscular média (CHCM) falsamente diminuídos, podendo comprometer o diagnóstico das anemias. Observaram que os valores do volume globular de sangue colhido com concentração de EDTA superior a 2 $\mathrm{mg} / \mathrm{ml}$ diminuíram significativamente.

Em humanos, acredita-se que a hemólise causada pelo EDTA seja oriunda de seu efeito osmótico sobre a membrana eritrocitária, cuja ação hemolítica é tão maior quanto maior for a concentração do anticoagulante (Blaxhall, 1973). Segundo Pinteric et al. (1975), o EDTA promove alterações na membrana dos eritrócitos de humanos, modificando sua morfologia e desencadeando o processo de hemólise osmótica. Vários autores relataram agregação de eritrócitos, plaquetas e, principalmente, leucócitos, associada à presença de EDTA (Deol et al., 1995; Lombarts et al., 1999).

O volume globular de sangue de aves e répteis colhidos com EDTA e Heparina foi similar. Entretanto, observaram-se maiores valores de VCM e de hemólise nas amos- tras colhidas com o primeiro anticoagulante (Hatting \& Smith, 1976). Segundo Fourie (1977), a Heparina é o anticoagulante mais adequado para a hematologia de pombos. A hemólise causada pelo EDTA foi observada em aves grou (Hawkey et al., 1983), corvo, peru e calau (Dein, 1986). Diferentemente, Thrall (2004) afirma que a heparina e o citrato podem ser utilizados nas análises hematológicas de aves. Entretanto, o EDTA apresenta a vantagem de preservar a morfologia e evitar aglomerados de leucócitos.

À semelhança do que ocorre com outras espécies aviárias, não há consenso quanto ao uso dos anticoagulantes na hematologia dos avestruzes. Kelly (1999), Spinu et al. (1999) e Ben Romdhane et al. (2000) utilizaram o EDTA, enquanto Raukar \& Simpraga (2005) fizeram uso do Citrato de Sódio e Palomeque et al. (1991) e Mushi et al. (1999) utilizaram a Heparina como anticoagulante de escolha para estudos hematológicos em avestruzes.

O efeito dos anticoagulantes sobre os parâmetros hematológicos de avestruzes, até o momento, não foi bem avaliado. Recentemente, Mafuvadze \& Erlwanger (2007), em estudo realizado com apenas oito avestruzes adultos, verificaram que amostras sanguíneas colhidas com heparina sódica apresentaram fragilidade osmótica e volume globular (VG) menores do que as colhidas com EDTA potássico. Na tentativa de explicar esses resultados, Mafuvadze \& Erlwanger (2007) hipotetizaram que o EDTA promove um aumento do tamanho dos eritrócitos de avestruzes e, consequentemente, maiores valores de VG. Neste mesmo estudo a conservação das amostras em temperatura ambiente não causou variação no valor do VG ao longo do tempo (0,5, 6 e 12 horas).

Para realização do presente estudo, considerou-se a escassez e contradição das informações da literatura supracitada, assim como o fato de que nos primeiros três meses pós-nascimento a concentração de hemoglobina fetal instável é mais elevada e que, neste período, a estrutiocultura sofre sérios prejuízos por causa da elevada taxa de mortalidade. Buscando melhor conhecer o efeito hematológicos dos anticoagulantes de rotina, decidiu-se testar a hipótese de que o volume, a integridade e a morfometria eritrocitária de avestruzes jovens diferem em amostras colhidas com EDTA sódico e Heparina sódica. 


\section{MATERIAL E MÉTODOS}

\section{Seleção dos animais}

Utilizaram-se 20 avestruzes com idades entre 7 e 62 dias, sendo oito fêmeas e doze machos, provenientes do incubatório do Curso de Medicina Veterinária da Universidade Estadual Paulista Julio de Mesquita Filho - UNESP - Campus de Araçatuba, São Paulo. Como critério de inclusão, consideraram-se apenas aves clinicamente saudáveis, no momento da coleta e, que, até 30 dias póscoleta apresentaram bom desenvolvimento corporal e nenhuma doença.

\section{Coleta das amostras}

Em coleta única, utilizando-se agulhas $(0,70$ x 25 mm) e seringas plásticas descartáveis, de cada ave, por flebocentese da jugular, obtiveram-se três mililitros de sangue, que foram distribuídos equitativamente em três diferentes frascos plásticos: Tubo E: contendo $20 \mu \mathrm{L}$ de solução aquosa de EDTA sódico ${ }^{4}$ a $10 \%$ (2 mg/mL sangue); Tubo H: contendo $2 \mu \mathrm{L}$ de Heparina sódica ${ }^{5}$ (10 U/ mL sangue) e Tubo SA: isento de anticoagulante.

\section{Análises laboratoriais}

As amostras foram mantidas refrigeradas $\left(4^{\circ} \mathrm{C}\right)$ até o momento de seu processamento ( 2 a 5 horas). O volume globular (VG) foi determinado pelo método de microhematócrito, conforme preconizado pelo National Committee for Clinical Laboratory Standards (NCCLS) (2000), utilizando-se tempo de centrifugação de cinco minutos e força de centrifugação de 11.827 g. A concentração de hemoglobina no soro e no plasma foi determinada pelo método da cianohemiglobina, utilizando-se um conjunto reativo de Drabkin comercial ${ }^{6}$ e leitura em espectrofotômetro ${ }^{7}$ com comprimento de onda de 540 nm. A coloração dos esfregaços sanguíneos foi realizada utilizando-se um conjunto de reativo panótico rápido comercial $^{8}$. A determinação morfométrica dos eritrócitos foi realizada com imagens obtidas por sistema de microscopia óptica ${ }^{9}$ (aumento 400X) acoplado à câmera de captura digital ${ }^{10}$ e analisadas por programa computadorizado de análise de imagem ${ }^{11}$ configurado para o cálculo automático de área e diâmetros máximo e mínimo de estruturas elípticas. Os parâmetros morfométricos foram estimados a partir das análises individuais de no mínimo 100 eritrócitos de cada amostra de sangue colhida com EDTA e Heparina, totalizando o mínimo de 2000 eritrócitos por tratamento.

\section{Análise estatística}

Todos os parâmetros foram previamente analisados quanto à normalidade pelo teste de Kolmogorov-Smirnov e homocedasticidade pelo teste de Bartlett. Conforme os resultados dessa análise inicial, a significância das diferenças de todos os parâmetros quanto ao sexo, ao VG, à área e aos diâmetros, entre os tratamentos EDTA e Heparina foi calculada pelo teste T de Student pareado ou de MannWhitney. Para a variável hemoglobina, realizaram-se os testes de Kruskall-Wallis e Dunn para a comparação múltipla de postos referentes aos tratamentos EDTA, Heparina e soro. Todas as análises estatísticas supracitadas foram realizadas em programa estatístico computadorizado ${ }^{12}$.

\section{RESULTADOS E DISCUSSÃO}

Todos os parâmetros analisados no presente estudo não apresentaram diferença estatística $(\mathrm{p}<0,05)$ quanto ao sexo, de modo que os dados foram analisados como um todo, levando-se em consideração apenas os tratamentos (EDTA e Heparina).

As concentrações de hemoglobina nos soros e em amostras com Heparina foram muito baixas e não diferiram significativamente (Tabela 1 ), indicando que a metodologia adotada na coleta e manipulação das amostras do experimento não promoveu perda significativa da integridade dos eritrócitos. Já o grau de hemólise indicado pela concentração de hemoglobina no plasma de amostras tratadas com EDTA foi superior $(p<0,01)$ ao verificado com amostras heparinizadas e do soro, independentemente da idade (Tabela 1). Esta maior concentração de hemoglobina no plasma colhido com EDTA evidencia o efeito hemolítico mais acentuado desse anticoagulante, quando comparado à Heparina, conforme descrito para as aves grou (Hawkey et al., 1983), corvo, peru e calau (Lumeij, 1986) e para avestruzes (Mafuvadze \& Erlwanger, 2007).

Independentemente da faixa etária dos avestruzes, a grande maioria (95\%) das amostras com EDTA apresentou VG maior do que as amostras tratadas com Heparina, sendo a maior diferença de 9\%. Diferentemente, Hatting \& Smith (1976) constataram valores de VG similares em amostras de aves e répteis colhidas com EDTA e Heparina. Entretanto, verificaram maiores valores de VCM e hemólise nas amostras colhidas com o primeiro anticoagulante. Embora o efeito hemolítico tenha sido maior em amostras com EDTA, o VG médio de amostras obtidas com esse sal foi significativamente superior aos com Heparina (Tabela 1). Este acha-

\footnotetext{
${ }^{4}$ EDTA Titriplex II GR for analysis, Merck, UK

${ }^{5}$ Heptar, 5000U, Eurofarma, São Paulo-SP

${ }^{5}$ Líquido de Drabkin, Newprov, Maringá-PR

Espectrofotômetro CELM E205D, São Paulo-SP

${ }^{8}$ Instant Newprov, Maringá-PR
}

\footnotetext{
${ }^{9}$ LEICA DMLS, Wetzlar, Germany

${ }^{10}$ LEICA DC 300, Heerbrugg, Schweiz

${ }^{11}$ Image-Pro ${ }^{\varpi}$ Plus, version 4.1, Media Cybernetics, Maryland, USA.

${ }^{12}$ SAS/Software, Statistical Analysis System Institute, 1997, USA
} 
Tabela 1. Média e desvios padrão do volume globular, do teor de hemoglobina plasmática e sérica e da morfometria dos eritrócitos de amostras de sangue de avestruzes colhidas com EDTA e Heparina.

\begin{tabular}{|c|c|c|c|c|}
\hline & EDTA & HEPARINA & Soro & P-value \\
\hline Hemoglobina (g/L) & $3,3 \pm 2,4^{\text {a }}$ & $0,1 \pm 0,3^{\mathrm{b}}$ & $0,2 \pm 0,2^{b}$ & $<0,01$ \\
\hline Volume globular (L/L) & $0,30 \pm 0,04^{\mathrm{a}}$ & $0,26 \pm 0,03^{b}$ & & 0,007 \\
\hline Área $\left(\mu \mathrm{m}^{2}\right)$ & $103,13 \pm 14,55^{a}$ & $99,67 \pm 14,72^{b}$ & & $<0,0001$ \\
\hline Diâmetro máximo $(\mu \mathrm{m})$ & $15,51 \pm 1,14^{a}$ & $15,42 \pm 1,64^{b}$ & & 0,03 \\
\hline Diâmetro mínimo $(\mu \mathrm{m})$ & $8,65 \pm 1,03^{\text {a }}$ & $8,98 \pm 1,17^{b}$ & & $<0,0001$ \\
\hline
\end{tabular}

* Letras não coincidentes na mesma linha indicam diferença significativa.

do aparentemente contraditório deve-se a um efeito osmótico do EDTA sobre os eritrócitos, ocasionando maior hemólise e aumento do volume das células mais resistentes não lisadas, conforme já descrito em humanos (Blaxhall, 1973; Pinteric et al. 1975) e em avestruzes (Mafuvadze \& Erlwanger, 2007). Segundo Jain (1993) o EDTA sequestra os íons $\mathrm{Ca}^{++}$responsáveis pela ativação da bomba de $\mathrm{Na}^{+}$e $\mathrm{K}^{+}$da membrana celular, permitindo a livre entrada de água para o interior da célula, promovendo seu intumescimento e consequente lise. Andreasen et al. (1997) e Mafuvadze \& Erlwanger (2007) afirmam que os eritrócitos dos avestruzes apresentam diferentes graus de fragilidade osmótica, causados pelas interações químicas com o EDTA e que este mecanismo de hemólise necessita de uma melhor investigação.

Mafuvadze \& Erlwanger (2007) hipotetizaram ainda que o EDTA causa aumento do tamanho dos eritrócitos de avestruzes. De fato, a média da área dos eritrócitos obtida com EDTA foi significativamente superior à obtida com Heparina (Tabela 1), sendo os menores eritrócitos (30-99 $\mu \mathrm{m}^{2}$ ) mais frequentes em amostras heparinizadas, enquanto os eritrócitos maiores (100-150 $\left.\mu \mathrm{m}^{2}\right)$ são mais numerosos em amostras com EDTA(Figura1).

Amostras colhidas com EDTA, quando comparadas às heparinizadas, apresentaram diferenças significativas quanto ao diâmetro maior e menor (Tabela 1), apresentando eritrócitos mais alongados. Essas diferenças devem ser consideradas em estudos que utilizam a morfometria

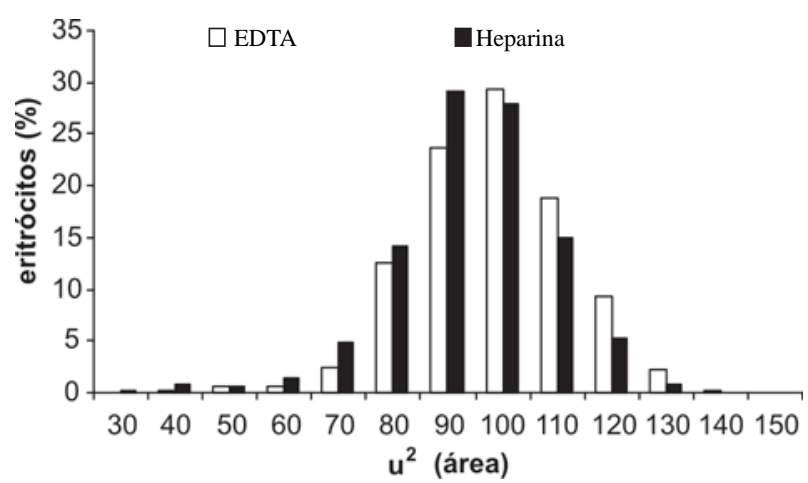

Figura 1. Freqüência de eritrócitos de acordo com a área, em amostras sangüíneas de avestruzes colhidas com EDTA e Heparina. eritrocitária para desenvolvimento de contadores automatizados e para investigação comparativa da evolução e adaptação das espécies.

O conjunto dos resultados obtidos indica que, para a adequada interpretação dos valores eritrocitários dos avestruzes, deve ser considerado o tipo de anticoagulante utilizado na colheita da amostra. Amostras colhidas com EDTA podem mascarar o diagnóstico de processos anêmicos, quando considerado o valor de referência de VG obtido com sangue heparinizado. Por outro lado, quando considerada a contagem eritrocitária, a amostra colhida com EDTA pode induzir a um falso diagnóstico de anemia, por causa do efeito hemolítico desse sal.

\section{CONCLUSÕES}

Para análises quantitativa e morfométrica dos parâmetros eritrocitários de avestruzes, a Heparina sódica (10 U/mL sangue) demonstrou ser um anticoagulante mais adequado do que o EDTA sódico (2mg/mL sangue).

\section{AGRADECIMENTOS}

Prof. Adj. Manoel Garcia Neto e Prof ${ }^{\text {a }}$. Ass. Dra. Valéria Maria Savoya da Silva, do Incubatório de Avestruzes do Departamento de Produção, Apoio e Saúde Animal do curso de Medicina Veterinária da Universidade Estadual Paulista Júlio de Mesquita Filho - UNESP - Campus de Araçatuba.

\section{REFERÊNCIAS}

Associação dos Criadores de Avestruz do Brasil (ACAB) (2005) Estrutiocultura. Disponível em: http://www.acab.org.br/ ?md=estatico\&pag=estrutiocultura/index Acessado em: 25 julho de 2007.

Andreasen CB, Andreasen JR \& Thomas JS (1997) Efects of hemolysis on serum chemistry analites in ratites. Veterinary Clinical Pathology, 26:65-171.

Ben Romdhane S, Romdane MN, Mhiri S, Ben Miled MA \& Kortas M. (2000) Les paramètres biochimiques et hematologiques chez l'autruche (Struthio camelus) dans un élevage tunisien. Révue de Médicine Vétérinaire, 3:231-238.

Blaxhall PC (1973) Error in haematocrit value produced by inadequate concentration of thilenediamine tetra-acetate. Journal of Fish Biology, 5:767-769. 
Dein FJ (1986) Clinical Avian Medicine and Surgery. Philadelphia, C. J. Harrison and L. R. Harrison. p.174-191.

Deol I, Hernandez AM \& Pierre RV (1995) Ethilenediaminetetraacetic acid - associated leukoagglutination. American Journal of Cinical Pathology, 103:338-340.

Fourie FR (1977) Effects of anticoagulants on the haematocrit, osmolarity and pH of avian blood. Poultry Science, 56:18421846.

Hatting J \& Smith EM (1976) Anticoagulants for avian and reptilian blood: heparin and EDTA. Pflugers Archives, 363:267269.

Hawkey C, Samour JH, Ashton DG, Hart MG, Cindery RN, Finch JM \& Jones DM (1983) Normal and clinical hematology of captive cranes (gruiformes). Avian Pathology, 12:73-84.

Jain NC (1993) Coagulation and its disorders. In: Jain NC (Ed.) Essentials of veterinary hematology. Philadelphia, Lea \& Fabiger. p. 82-104

Kelly MP (1999) Psittacine blood and hematology, In: Kelly MP (Ed.) Basics for the veterinary praticioner. Disponível em: < http://www.vet.uga.edu/vpp/ivcvm/1999/phillips/index.php >. Acessado em: 3 maio de 2007.

Lombarts AJPF, Zijstra JJ, Peters RHM, Thomasson CG \& Franck PFH (1999) Accurate platelet counting in an insidious case of pseudothrombocytopenia. Clinical and Chemical Laboratory Medicine, 37:1063-1066.

Lumeij JT (1986). Avian clinical biochemistry. In: Kaneko JJ, Harvey JW \& Bruss ML (Eds.) Clinical biochemistry of domestical animals. Philadelphia, C. J. Harrison and L. R. Harrison. p.858.

Mafuvadze B \& Erlwanger KH (2007) The effect of EDTA, heparin and storage on the erythrocyte osmotic fragility, plasma osmolality and haematocrit of adult ostriches (Struthio camelus). Veterinarski Arhiv, 77:427-434.
Mushi EZ, Binta GM, Chabo RG, Isa JFW \& Kapaata RW (1999) Selected hematologic values of farmed ostriches (Struthio camelus) in Botswana. Journal Veterinary Diagnostic Investigation, 11:372-374.

National Committee for Clinical Laboratory Standards (NCCLS) (2000) Procedure for determining packed cell volume by the microhematocrit method; Approved standard. $3^{\text {a }}$ ed. document H7-A3. Disponível em: < http://www.clsi.org/source/orders/free/ h7-a3.pdf > . Acessado em: 5 maio de 2007.

Palomeque J, Pintó D \& Viscor G (1991) Hematologic and blood chemistry values of the masai ostrich (Struthio camelus). Journal of Wildlife Diseases, 271:34-40.

Pennock CA \& Jones KW (1966) Effect of ethylene-diaminetetracetic-acid (dipotassium salt) and heparin on the estimation of packed cell volume. Journal of Clinical Pathology, 19:196199.

Pinteric L, Manery JF, Chaudry IH \& Madalpallimattam G (1975) The effect of EDTA, cations, and various buffers on the morphology of erythrocyte membranes: an electronmicroscopic study. Blood, 45:709-724.

Raukar J \& Simpraga M (2005) Haematological parameters in the blood of one day old ostriches. Israel Journal of Veterinary Medicine, 60:112-116.

Spinu M; Spinu O \& Degen AA (1999) Haematological and imunological variables in a domesticated and wild subspecies of ostrich (Struthio camelus). British Poultry Science, 40:613618.

Thrall MA (2004) Hematology of birds. In: Thrall MA (Ed.) Veterinary hematology and clinical chemistry. Philadelphia, Lea \& Fabiger. p.225-258. 